\title{
Ions for LHC: Beam Physics and Engineering Challenges
}

\author{
M.E. Angoletta, V. Baggiolini, A. Beuret, A. Blas, J. Borburgh, H.H. Braun, C. Carli, M. Chanel, \\ A. Fowler, S. Gilardoni, M. Gourber-Pace, S. Hancock, C.E. Hill, M. Hourican, J.M. Jowett, \\ K. Kahle, D. Küchler, E. Mahner, D. Manglunki, M. Martini, S. Maury, M. Paoluzzi, \\ J. Pasternak, F. Pedersen, U. Raich, C. Rossi, J.P. Royer, K. Schindl, R. Scrivens, \\ L. Sermeus, E. Shaposhnikova, G. Tranquille, M. Vretenar, T. Zickler \\ CERN, Geneva, Switzerland
}

\begin{abstract}
The first phase of the heavy ion physics program at the LHC aims to provide lead-lead collisions at energies of $5.5 \mathrm{TeV}$ per colliding nucleon pair and ion-ion luminosity of $10^{27} \mathrm{~cm}^{-2} \mathrm{~s}^{1}$. The transformation of CERN's ion injector complex (Linac3-LEIR-PS-SPS) presents a number of beam physics and engineering challenges, which are described in this paper. In the LHC itself, there are fundamental performance limitations due to various beam loss mechanisms. To study these without risk of damage there will be an initial period of operation with a reduced number of nominal intensity bunches. While reducing the work required to commission the LHC with ions in 2008, this will still enable early physics discoveries.
\end{abstract}

Presented at PAC 2005, Knoxville, USA, May 16-20, 2005

CERN,

CH-1211 Geneva 23,

Switzerland

Geneva, June 2005 


\title{
IONS FOR LHC: BEAM PHYSICS AND ENGINEERING CHALLENGES
}

\author{
M.E. Angoletta, V. Baggiolini, A. Beuret, A. Blas, J. Borburgh, H.H. Braun, C. Carli,
} M. Chanel, A. Fowler, S. Gilardoni, M. Gourber-Pace, S. Hancock, C.E. Hill, M. Hourican, J.M. Jowett, K. Kahle, D. Küchler, E. Mahner, D. Manglunki, M. Martini, S. Maury, M. Paoluzzi, J. Pasternak, F. Pedersen, U. Raich, C. Rossi, J.P. Royer, K. Schindl, R. Scrivens, L. Sermeus, E. Shaposhnikova, G. Tranquille, M. Vretenar, T. Zickler, CERN, Geneva, Switzerland

\section{Abstract}

The first phase of the heavy ion physics program at the LHC aims to provide lead-lead collisions at energies of 5.5 TeV per colliding nucleon pair and ion-ion luminosity of $10^{27} \mathrm{~cm}^{-2} \mathrm{~s}^{-1}$. The transformation of CERN's ion injector complex (Linac3-LEIR-PS-SPS) presents a number of beam physics and engineering challenges, which are described in this paper. In the LHC itself, there are fundamental performance limitations due to various beam loss mechanisms. To study these without risk of damage there will be an initial period of operation with a reduced number of nominal intensity bunches. While reducing the work required to commission the LHC with ions in 2008, this will still enable early physics discoveries.

\section{OVERVIEW}

The accelerator chain for LHC ion operation [1] and major hardware upgrades needed are sketched in Fig. 1.

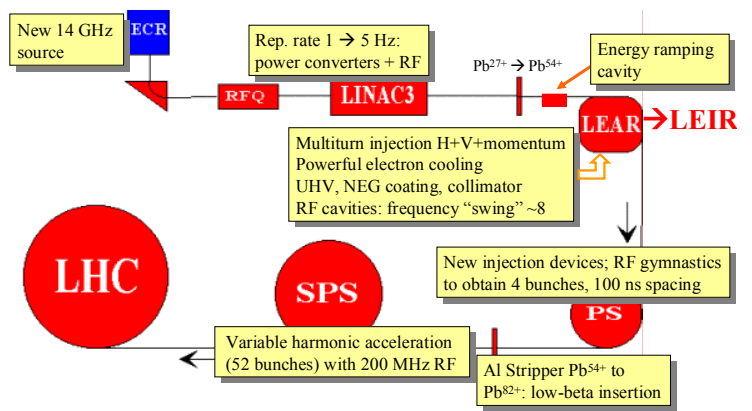

Figure 1: Hardware upgrades in the LHC injector chain.

In the nominal scheme, the injector chain provides the LHC with 592 bunches of $9 \times 10^{7} \mathrm{~Pb}^{82+}$ ions per ring. The beam sizes and bunch length at SPS extraction and at collision in the LHC are the same as for protons, resulting in a lead-lead luminosity of $10^{27} \mathrm{~cm}^{-2} \mathrm{~s}^{-1}$. The nominal lead beam may be subject to limitations in the injectors and in the LHC [2]. As these effects are not easy to predict accurately, it is prudent to start with a beam whose characteristics allow the limitations to be explored with reduced risk. The "early ion scheme" (Table 1) has fewer bunches (only 60 per LHC ring with $1.35 \mu \mathrm{s}$ bunch spacing) with the same bunch intensity and $\beta^{*}=1 \mathrm{~m}$ (instead of $0.5 \mathrm{~m}$ ), yielding a luminosity of $5.10^{25} \mathrm{~cm}^{-2} \mathrm{~s}^{-1}$ suitable for the first year.

\section{LINAC3 UPGRADE}

A new electron cyclotron resonance (ECR) source,
Table 1: Beam parameters along the accelerator chain for "early LHC ion operation"

\begin{tabular}{|c|c|c|c|c|c|c|}
\hline \multirow[b]{2}{*}{ Output energy } & \multicolumn{6}{|c|}{ ECR Source $\longrightarrow$ Linac $3 \longrightarrow$ LEIR $\longrightarrow$ PS $\underset{4.2}{\longrightarrow}$ sPS $\overrightarrow{12}$ LHC } \\
\hline & $2.5 \mathrm{KeV} / \mathrm{n}$ & $4.2 \mathrm{MeV} / \mathrm{n}$ & $72.2 \mathrm{MeV} / \mathbf{n}$ & $5.9 \mathrm{GeV} / \mathbf{n}$ & $177 \mathrm{GeV} / \mathbf{n}$ & $2.76 \mathrm{TeV} / \mathrm{n}$ \\
\hline $2{ }^{2} \mathrm{~Pb}$ charge state & $27+$ & $27+54+$ & $54+$ & $54+\stackrel{i}{\rightarrow} 82+$ & $82+$ & $82+$ \\
\hline Output $\mathrm{B} \rho \quad[\mathrm{Tm}]$ & & $2.28 \geqslant 1.14$ & 4.80 & $86.7 \rightarrow 57.1$ & 1500 & 23350 \\
\hline number of bunches & & & 1 (1/8 of $\mathrm{PS})$ & $\mathrm{i}$ & 4,2 & $62 /$ ring \\
\hline ions/pulset, 2 & $910^{\circ}$ & $1.1510^{\circ}$ & $2.2510^{3}$ & $1.210^{8}$ & $\leq 3.610^{8}$ & $4.310^{9}$ \\
\hline ions/LHC bunch & $910^{9}$ & $1.1510^{9}$ & $2.2510^{8}$ & $1.210^{8}$ & $910^{7}$ & $710^{7}$ \\
\hline bunch spacing [ns] & & & & & 1350 & 1350 \\
\hline$\varepsilon^{*}$ (nor. rms) $[\mu \mathrm{m}]^{3}$ & 0.10 & 0.25 & 0.7 & 1.0 & 1.2 & 1.5 \\
\hline$\varepsilon$ (phys.rms) $[\mu \mathrm{m}]^{3}$ & 50 & 2.5 & 1.75 & 0.14 & 0.0063 & 0.0005 \\
\hline Repetition time [s] & & 2.4 & 2.4 & 2.4 & 18 & s'rill/ring \\
\hline total bunch length $[\mathrm{ns}]$ & & & 200 & 3.9 & 1.65 & 1 \\
\hline
\end{tabular}

designed and built at CEA/Grenoble [3], operating in "afterglow mode" at $14.5 \mathrm{GHz}$, will deliver the required current of $200 \mathrm{e} \mu \mathrm{A} \mathrm{Pb}^{27+}$. Compared to the older source (ECR4), it has increased transverse confinement by a stronger permanent hexapole; a strong longitudinal field (up to $1.4 \mathrm{~T}$ ); a larger plasma chamber; RF couplers for both 14.5 and $18 \mathrm{GHz}$ microwaves and the possibility to install two micro-ovens. It performed well in first tests: $\sim 500$ e $\mu \mathrm{A}$ of $\mathrm{O}^{6+}$ and $\sim 40 \mathrm{e} \mu \mathrm{A}$ of $\mathrm{Ar}^{12}$. Pulsed power converters for magnets to LEIR are being upgraded for the operation at $5 \mathrm{~Hz}$. A dedicated ramping cavity, installed directly downstream of the Linac3 stripper, varies the beam momentum by $\pm 0.4 \%$ along the pulse by a linear modulation of the cavity phase by $\pm 40^{\circ}$. The cavity is a $100 \mathrm{MHz}$ 4-gap spiral-loaded RF resonator, capable of delivering a $250 \mathrm{kV}$ RF voltage to the beam. The debunching cavity placed $11 \mathrm{~m}$ downstream has to be phase-modulated in the same range to compensate for the change in ion time of flight and to minimise momentum spread. The ramping system has been successfully tested with beam in static mode; dynamic tests will be done during the start-up.

\section{UPGRADE OF LEAR TO LEIR}

\section{LEIR Cycle}

The role of LEIR is to transform a series of long $(\sim 200 \mu$ s), low-intensity ion pulses from Linac3 into short ( $200 \mathrm{~ns})$, high-brightness bunches. Each $\mathrm{Pb}^{54+}$ linac pulse is injected with $70 \%$ efficiency in horizontal, vertical and longitudinal phase space using and inclined electrostatic septum and by energy ramping. On a 4.2 MeV/n plateau in LEIR, the electron cooler strongly reduces the phase space volume of the beam in less than $400 \mathrm{~ms}$ and decelerates it into a stack sitting slightly 
inside the central orbit. For the early beam only one $\mathrm{Pb}^{54+}$ linac pulse is injected (4 for the nominal), cooled, adiabatically captured on $\mathrm{h}=1$ and accelerated to $72 \mathrm{MeV} / \mathrm{n}$. For the early beam the LEIR cycle is shortened to $2.4 \mathrm{~s}$.

\section{Transfer Lines}

The $4.2 \mathrm{MeV} / \mathrm{n}$ beam from Linac3 and the $72 \mathrm{MeV} / \mathrm{n}$ one extracted towards the PS share $\sim 60 \mathrm{~m}$ of common transfer line in which they travel in opposite directions. Thus, all magnets must be laminated. Power converters of most bending magnets are bipolar; those for quadrupoles are monopolar due to appropriate optics.

\section{Layout and Lattice}

LEIR is the same shape as LEAR [4] but its optics is new and flexible enough to optimize multi-turn injection and electron cooling. The injection system comprises a DC magnetic septum, a new inclined electrostatic septum, and four fast bumper magnets incorporating ceramic chambers and providing bump collapse times of 120 $500 \mu$ s for multi-turn injection. All correction dipoles and multipoles are recovered from LEAR. To extract the beam at $4.8 \mathrm{Tm}$, the former LEAR injection kicker modules are powered by new pulse generators and a new pulsed septum provides $0.8 \mathrm{~T}$ through a thin-walled stainless steel vacuum chamber. All elements have to withstand bake-out at $300^{\circ} \mathrm{C}$.

\section{Electron Cooling}

The electron cooler was constructed in collaboration with BINP, Russia. After successful tests in Novosibirsk, it was delivered to CERN at the end of 2004. The state-ofthe-art design [5] incorporates many of the latest ideas in electron cooling technology such as a convex cathode for the generation of intense $(>500 \mathrm{~mA})$ electron beams at low energy and the possibility to vary the dimension and density distribution of the electron beam. The latter is needed to rapidly cool the newly injected ions without excessive recombination with the cooled ion stack.

Following detailed measurement of the magnetic field, the cooler is now being prepared for installation. First tests with an electron beam will start at the beginning of June and cooling tests with an ion beam in September.

\section{Vacuum}

Heavy-ion induced molecular desorption, currently observed in several particle accelerators world-wide, will be critical for the operation of the LEIR vacuum system in the $10^{-12}$ Torr range. Electron-capture from the residual gas will cause lost $\mathrm{Pb}^{53+}$ ions to hit the vacuum chambers and desorb molecules. Large desorption yields of $\sim 10^{4}$ molecules $/ \mathrm{Pb}^{53+}$ ion were measured for bare $316 \mathrm{LN}$ stainless steel chambers [6]. Following investigations at Linac3 [7], methods to reduce large pressure rises were implemented in LEIR [1]: non-evaporable getter coating (TiZrV) of most vacuum chambers to obtain very clean surfaces after in situ bakeout $\left(300^{\circ} \mathrm{C}\right)$ and low dynamic gas loads under lead ion bombardment, installation of gold-coated collimators to control ion losses [8] and beam scrubbing to improve the dynamic pressure by continuous ion bombardment.

\section{$R F$ and feedback systems}

Two new large-bandwidth $(0.35-5 \mathrm{MHz}$ without any tuning) cavities based on Finemet ${ }^{\circledR}$ high-permeability magnetic alloy have been built in collaboration with KEK Acceleration at LEIR's moderate ramp rate requires an RF voltage of less than $4 \mathrm{kV}$, keeping the amplifier power to a reasonable $60 \mathrm{~kW}$. The available frequency range covers lead ions at harmonic $\mathrm{h}=2$ or $\mathrm{h}=1$ and keeps open the option of lighter ions at a later date. The cavities are installed and being commissioned [9].

LEIR is the first CERN accelerator to be equipped with an all-digital beam control and cavity servoing system for the low-level RF. A prototype has been tested successfully in the PS Booster [10]. Following the LEIR commissioning, a plan will be finalized to migrate to the same technology PS, PSB and AD.

\section{Other systems}

Most of the 164 power converters in LEIR and its transport lines are being rebuilt or recuperated. They are based on thyristor or switch-mode technologies; there are also pulsed power converters as well as HV supplies for $\mathrm{RF}$ and electron cooling. Commissioning of some power converters has started.

Beam diagnostic devices are mostly recovered from LEAR but have to be adapted to ions as well as to new standards for electronics and control. Of particular importance are the DC current transformer $(2 \mu \mathrm{A}$ to $50 \mathrm{~mA}$ ), the Schottky pick-ups (to measure the emittance and energy spread of coasting beam) and beam ionization profile monitors (to observe beam dimensions during cooling). Some devices have been installed, and others, like the transformer, are being manufactured.

LEIR is the first circular machine that will make use of the unified control infrastructure developed for LHC and ultimately for all the CERN accelerators. This system is capable of so-called "multicycling", which will allow the ion complex to be tested on a cycle-to-cycle basis in parallel with beam production for physics. The development of the LEIR specific application programs running on top of the standard infrastructure has started.

\section{PS TO SPS}

The beam is injected into the PS from LEIR by two pulsed bumper magnets, an upgraded kicker magnet and a new pulsed septum. The two bunches fill $1 / 8$ of the PS, which in turn has to provide 4 bunches to the SPS. This is achieved by a rather elaborate procedure [1] involving harmonic changes and bunch splitting and making use of the RF systems $(3-10,80 \mathrm{MHz})$ that produce the LHC proton beam. After extraction from the PS, the $\mathrm{Pb}^{54+}$ beam is fully stripped to $\mathrm{Pb}^{82+}$ by a $0.8 \mathrm{~mm}$ aluminum foil. In order to reduce the emittance blow-up [11] caused by 
Coulomb scattering to values compatible with the tight emittance budget (Table 1), the stripper foil must be at low $\beta$. Four new quadrupoles and six new power converters are needed to reduce $\beta$ by a factor 5 in the PSSPS line. For the proton beams which also pass through this line, the new quadrupoles should ideally be set to zero gradients and their residual field blows up the proton emittance by less than $0.1 \%$.

In the early ion scheme, up to 4 PS batches, each one providing only one LHC bunch, are injected into the SPS on a $7.2 \mathrm{~s}$ injection plateau at $5.9 \mathrm{GeV} / \mathrm{n}$. Analysis of the nominal acceleration cycle for LHC ions in the SPS has shown the possibility of using a $3 \mathrm{~s}$ instead of the $10 \mathrm{~s}$ acceleration cycle proposed in the past and justified for the lower injection energy. The new magnetic cycle with a gently rising initial part of the ramp should reduce the LHC filling time. At an intensity of $1.2 \times 10^{8} \mathrm{~Pb}^{82+}$ ions/bunch, the space-charge tune shift and calculated intra-beam scattering growth times are acceptable.

\section{LHC MAIN RINGS}

Lead ion collisions will be provided to 3 LHC experiments. To minimise the time needed to switch the LHC main rings from their $p-p$ collider mode, the magnetic cycle used for lead ions will be very similar, with the exception that it will be necessary to squeeze a third IP (ALICE) to $\beta^{*}=1 \mathrm{~m}$ in the early ion scheme. An even faster transition to $\mathrm{Pb}-\mathrm{Pb}$ collisions with luminosity of $5.10^{24} \mathrm{~cm}^{-2} \mathrm{~s}^{-1}$ may be feasible during the initial proton commissioning, when all IPs will collide with $\beta^{*}$ at injection values. Limitations of the dynamic range of key beam instrumentation (BPMs and current monitors in particular) mean that the single bunch currents are the same in the nominal and early schemes. Many limiting effects, particularly those leading to emittance growth [2], will therefore be present in full measure, although none are now expected to be insuperable. Only later, in the nominal scheme, will total current and luminosity be limited by beam losses that may potentially quench magnets [2,12]. Continuous beam losses proportional to luminosity are caused by electromagnetic interactions in collisions; losses proportional to beam current by the inefficiency of the collimation system. Studies with the early beam will help to resolve the uncertainties concerning their severity.

\section{TENTATIVE SCHEDULE}

In order to meet the deadline for the lead collisions in the LHC, April 2008, the project has to meet the milestones as compiled in Table 2. Progress is satisfactory for most of the system; the schedule is ambitious and optimistic concerning LEIR installation. Any delay here is incompatible with commissioning LEIR with beam in August 2005. In 2006, in order to help the PS start-up with the large diversity of beams after the long 18-months shutdown, LEIR will stop at the end of March and restart for the PS commissioning as from September.
Table 2: Tentative schedule

\begin{tabular}{|c|c|c|c|}
\hline & \begin{tabular}{|l|} 
hardware \\
test
\end{tabular} & $\begin{array}{l}\text { Start with } \\
\text { beam }\end{array}$ & Problems \\
\hline Source, Linac3 & Feb. 2005 & Mar 2005 & New source \\
\hline LEIR inj. line & Mar 2005 & Jun 2005 & \\
\hline LEIR ring & Apr. 2005 & Aug. 2005(?) & $\begin{array}{l}\text { LEIR conversion completed? } \\
\text { Running-in through winter to } \\
\text { March } 2006\end{array}$ \\
\hline $\mathrm{PS} / \mathrm{TT} 2$ & Feb. 2006 & Sept. 2006(?) & Stop from April-August 2006 \\
\hline SPS & & $\begin{array}{l}\text { late } 2006 \\
\text { spring } 2007\end{array}$ & $\begin{array}{l}\text { SPS experts commissioning } \\
\text { LHC ring in } 2007\end{array}$ \\
\hline LHC & & $\begin{array}{l}\text { from Apr. } \\
2008(?)\end{array}$ & $\begin{array}{l}\text { Physics with the early beam } \\
\text { in LHC }\end{array}$ \\
\hline
\end{tabular}

\section{CONCLUSION}

The baseline LHC ion program foresees lead-lead collisions with reduced luminosity (early ion scheme) in 2 or 3 experiments in 2008. The early beam scenario is just the first step, so even before the commissioning of the injectors is finished, studies on the nominal beam have to be pursued as from 2007 in order to have nominal LHC luminosity in 2009. The task of the injectors is facilitated by the early beam scheme. LEIR installation is progressing satisfactorily and the challenge to start the beam commissioning in August 2005 until March 2006 is ready to be taken up.

\section{REFERENCES}

[1] LHC Design report Vol. III, Chapters 32-38 and Vol. I, Chapter 21, CERN-2004-003.

[2] J.M. Jowett et al., "Limits to the performance of the LHC with Lead Ions", Proc. EPAC 2004, MOPLT020.

[3] C.E. Hill et al, "GTS-LHC: a new source for the LHC ion injector chain”, AIP Conf. Proc. 749, p.127, 2005

[4] M. Chanel, "LEIR: The Low Energy Ion Ring", Proc. EPAC2002, THPLE074.

[5] G. Tranquille, "Specification of a new Electron Cooler for the Low Energy Accumulator Ring, LEIR", Proc. of COOL03, Japan, May, 2003.

[6] E. Mahner et al, "Molecular desorption of stainless steel vacuum chambers irradiated with $4.2 \mathrm{MeV} / \mathrm{u}$ lead ions", Phys. Rev. ST-AB, Vol. 6, 013201(2003).

[7] E. Mahner et al., "Ion-stimulated gas desorption yields of coated $(\mathrm{Au}, \mathrm{Ag}, \mathrm{Pd})$ stainless steel vacuum chambers irradiated with 4.2 MeV/u lead ions", CERN AT/2003-6 VAC.

[8] C. Bal, C. Carli, M. Chanel, E. Mahner, J. Pasternak, "A collimation scheme for ions changing charge state in the LEIR", FPAE070, this Conference.

[9] M. Haase et al., "The LEIR RF System", WPAT018, this conference.

[10] M.E. Angoletta et al., "Beam Tests of a New Digital Beam Control System for the CERN LEIR Accelerator", WPAT019, this conference.

[11] M. Martini,"Compatibility of New TT2 Low-Beta Insertion Required for Ion Beams with the Proton Beams", to be published.

[12] J.M. Jowett et al., "Heavy-ion Luminosity Limit from Bound-free Production in the LHC", TPAP012, this conference. 